\title{
Jean-Jacques Rousseau, Lettres philosophiques
}

\section{Paola Sosso}

\section{(2) OpenEdition}

\section{Journals}

\section{Edizione digitale}

URL: http://journals.openedition.org/studifrancesi/36193

DOI: 10.4000/studifrancesi.36193

ISSN: 2427-5856

\section{Editore}

Rosenberg \& Sellier

\section{Edizione cartacea}

Data di pubblicazione: 1 juillet 2005

Paginazione: 168-169

ISSN: 0039-2944

\section{Notizia bibliografica digitale}

Paola Sosso, "Jean-Jacques Rousseau, Lettres philosophiques», Studi Francesi [Online], 145 (XLIX | I) | 2005, online dal 30 novembre 2015, consultato il 19 avril 2021. URL: http://journals.openedition.org/ studifrancesi/36193; DOI: https://doi.org/10.4000/studifrancesi.36193

\section{Questo documento è stato generato automaticamente il 19 avril 2021.}

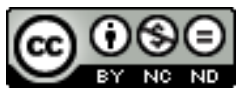

Studi Francesi è distribuita con Licenza Creative Commons Attribuzione - Non commerciale - Non opere derivate 4.0 Internazionale. 


\title{
Jean-Jacques Rousseau, Lettres philosophiques
}

\author{
Paola Sosso
}

\section{NOTIZIA}

JEAN-JACQUES ROUSSEAU, Lettres philosophiques. Édition établie, présentée et annotée par Jean-François PERRIN, Paris, Le Livre de Poche, 2003, pp. 571.

1 Negli ultimi anni la Correspondance di Rousseau ha occupato uno spazio sempre più ampio all'interno degli studi critici, che hanno ribaltato il giudizio negativo formulato da Daniel Mornet all'inizio del secolo scorso, evidenziando l'importanza delle lettere del Ginevrino per la comprensione del suo pensiero. Rousseau, come testimoniano le Confessions, aveva una concezione organica dei rapporti tra la sua corrispondenza e i suoi testi letterari e filosofici: certo non si può dire che l'epistolario sia stato dimenticato, come dimostrano l'edizione Moultou-Du Peyrou (1782), le edizioni MussetPathay e Streckeisen-Moultou dell'Ottocento e, in tempi più recenti, l'edizione Raymond-Gagnebin (1959-1995), la curatissima edizione Leigh (1965-1996) e ancora, per quanto concerne i testi a carattere filosofico, l'edizione Gouhier del 1974. La presente raccolta, con funzione divulgativa, mira tuttavia a far meglio conoscere al grande pubblico scritti per lo più poco noti, relegati talvolta in appendice alle opere maggiori o, addirittura, studiati solo dagli specialisti. Non può non stupire il titolo dell'antologia, Lettres philosophiques, per un pensatore che preferiva essere chiamato ours piuttosto che philosophe: tuttavia, come spiega il curatore, il termine "filosofico" vuole qui indicare il carattere "morale", "spirituale" e "politico" dei quarantasei testi raccolti. L'antologia copre un arco di tempo di ventinove anni, dalla lettera a M. de Conzié (1742) in cui Jean-Jacques, ospite alle Charmettes, si oppone con una dialettica immatura a un avversario di Pope che non è ancora Voltaire, fino alla prima delle Lettres sur la botanique (1771). Il percorso appare interessante e abbastanza ricco, perché accanto agli scritti celebri, come la lettera a Voltaire sulla Provvidenza, la "Lettre à Philopolis", le "Lettres morales", le lettere a Malesherbes, vi sono lettere meno conosciute che fino ad 
oggi trovavamo solo nella prestigiosa edizione della Correspondace complète di R. Leigh. Compare in questi testi un Rousseau per così dire "in veste privata", che risponde a una duchessa inglese appassionata di botanica, a giovani corrispondenti, di sesso maschile o femminile (tra cui Henriette), in preda all'inquietudine e all'angoscia, al principe de Würtemberg che intende educare sua figlia secondo i principi dell'Emile, o ancora ad alcuni lettori desiderosi di meglio comprendere il suo pensiero. La presente edizione si rifà principalmente all'edizione Leigh, ma tiene conto anche dei contributi offerti dall'edizione Raymond-Gagnebin e dall'edizione Gouhier. L'ortografia è stata modernizzata per favorire l'accesso a un più vasto pubblico, mentre le note, complete $\mathrm{e}$ precise, sono costruite a partire da svariati studi. In appendice si trova indicata la fonte manoscritta principale di ogni lettera e i dati riguardanti la prima pubblicazione. Semplice e agevole per i non specialisti il glossario, seguito da una rapida bibliografia che elenca alcuni testi fondamentali. 